\title{
UN ASPECTO DEL MODELADO DE LA CUENCA DE BUSOT: LOS GLACIS
}

\author{
Margarita Box Amorós
}

\section{SÍNTESIS GEOLÓGICA}

Introducción.- El área de estudio se encuentra en una posición relativamente conflictiva de cara a su ubicación dentro de las unidades béticas. Es de todos modos clara su pertenencia al dominio externo de las mismas, si bien la controversia se establece a la hora de enclavar dicha zona en el dominio Subbético o Prebético. Conviene, pues, comenzar por advertir que se trata de un sector donde ambos conjuntos entran en contacto, aunque las investigaciones realizadas por los geólogos han puesto de manifiesto la naturaleza prebética de este área.

La división del dominio externo de las cordilleras Béticas en Subbético y Prebético responde a los primeros trabajos de P. FALLOT ${ }^{1}$. Sin embargo, posteriormente, los investigadores integrados en su escuela, como DURAND DELGA y GARCÍA RODRIGO, propusieron la subdivisión del Prebético del área de Alicante en dos partes: Prebético interno y externo $^{2}$, mientras que FALLOT hacía extensiva la denominación de Prebético a todo el sector, si bien el mismo aparece con bastante frecuencia enmascarado por la existencia de sedimentos depositados posteriormente al plegamiento o perforado por extrusiones diapíricas, por [182] ello, y según FALLOT, es posible la presencia de elementos subbéticos cuya existencia es difícil de advertir. La razón fundamental de esa subdivisión está en el hecho de que el dominio Prebético externo engloba una masa de terrenos cretácicos, sobre todo de los pisos superiores, mientras que, por el contrario, el conjunto Prebético interno y Subbético está dominado por el roquedo del bajo Terciario o Eógeno, pese a que el despliegue tectónico bético ha implantado un aparente desorden ${ }^{3}$.

De todos modos, no se trata de una identidad total ni puede hacerse por una serie de razones una simple asimilación entre el dominio Prebético interno y el Subbético, ya que, si bien desde un punto de vista estratigráfico la similitud es bastante completa, como ya se ha indicado, desde un punto de vista estructural sus aspectos son diferentes, ya que en el caso del Prebético interno las unidades son autóctonas o parautóctonas, aun admitiendo un cierto desplazamiento NNW, mientras que la característica fundamental de las estructuras subbéticas es la aloctonía. De ordinario, se admite como Prebético externo las formaciones al norte de Alcoy-Bernia, Prebético interno al norte de Monóvar-Alicante, marcando la denominación de Subbético para el conjunto situado al norte de Crevillente-Elche ${ }^{4}$. En consecuencia, la zona de estudio quedaría integrada en el dominio Prebético interno con arreglo a esta delimitación.

\footnotetext{
${ }^{1}$ FALLOT, P.: Estudios geológicos en la zona subbética entre Alicante y el río Guadiana Menor. Instituto «Lucas Mallada» (C.S.I.C.) Madrid, 1945 p. 615 y ss.

${ }^{2}$ ROSSELlÓ VERGER, V. M. y BERNABÉ MAESTRE, J. M.: «La Montaña y sus valles: un dominio subhúmedo» en LÓPEZ GÓMEZ, A y ROSSELLÓ VERGER, V. M.: Geografía de la provincia de Alicante. Excma. Dip. Prov. de Alicante, 1978 p. 77.

${ }^{3}$ ROSSELLÓ VERGER, V. M.: «Un variado litoral» en LÓPEZ GÓMEZ, A. y ROSSELLÓ VERGER, V. M.: Geografia de la provincia de Alicante. Excma. Dip. Prov. de Alicante. Alicante, 1978 p. 6.

${ }^{4}$ ROSSELlÓ VERGER, V. M. y BERNABÉ MAESTRE, J. M. Op. cit. p. 77.
} 
Sin embargo, RODRÍGUEZ ESTRELLA ${ }^{5}$ hace una diferenciación del Prebético en tres sectores: Prebético externo, Prebético interno y Prebético meridional, en función de la existencia de diferencias estratigráficas y tectónicas substanciales de unos puntos a otros. El área de estudio, según dicho autor, quedaría englobada dentro del Prebético meridional, cuyo límite septentrional vendría definido, en la actualidad, por una línea que pasa por el $\mathrm{W}$ de las sierras Seca, Mariasnal, Huebra y Tobar. Dicho límite se ve interrumpido y desplazado hacia el E por la falla de la Línea Eléctrica y continúa al N de las sierras de Ascoy, Larga, Carche, Salinas, Peñarrubia, Mariola, Cantalar, Alfarada, Tierra Nueva y Almirante. El límite meridional lo constituye la zona subbética y en su extremo más oriental la depresión de Crevillente-Elche.

En líneas generales, en el conjunto Prebético, no aflora el zócalo premesozoico [183] y la cobertera comprende terrenos desde el Mesozoico hasta el Terciario (Mioceno inferior inclusive $)^{6}$ aunque cabría reseñar aquí que en la zona de estudio afloran, además de los materiales anteriormente citados, otros pertenecientes al roquedo neógeno y que, incluso, los materiales de los períodos Eoceno y Oligoceno que forman una estructura de tipo Flysch en la zona subbética, en este sector también aparecen con este tipo de formación, si bien se trata aquí más que de una estructura tipo Flysch propiamente, de un «pseudo-flysch».

Las facies son continentales y marinas de aguas someras: calizas, dolomías, margas y areniscas son las rocas más abundantes. En conjunto, la serie prebética presenta las características propias de un dominio paleogeográfico cuyas condiciones serían, bastante aproximadamente, las de una plataforma. La estructura tectónica de la zona prebética es, relativamente, sencilla, tratándose fundamentalmente de una cobertera despegada del zócalo a nivel del Trías y plegada, en líneas generales, con poca intensidad ${ }^{7}$.

Evolución geológica.- La sedimentación del área se inicia en la Era Secundaria, concretamente en el período Triásico, en un medio marino que evoluciona hasta convertirse en una cuenca lacustre. El tipo de facies predominante es el que RODRÍGUEZ ESTRELLA ${ }^{8}$ denomina de «interior de cuenca». Se trata, aquí, de un Triásico con predominio de materiales del Keuper, cuyos afloramientos presentan, normalmente, forma de bandas alargadas coincidentes, por lo general, con líneas de fractura, es decir, el emplazamiento de estos materiales hay que asociarlo a un fenómeno de extrusiones diapíricas en las zonas de distensión estructural y que, en este caso, se materializarían en la existente a lo largo del Río Montnegre o Seco. La potencia de la sedimentación es imposible de precisar, al igual que en el resto del área prebética, ya que se trata de afloramientos diapíricos y el muro nunca se ha observado ${ }^{9}$. La litofacies predominante es la de arcillas y areniscas rojas, abundando también los yesos y anhidritas.

La aparición de materiales jurásicos en superficie hay que relacionarla, también, con fenómenos extrusivos en aquellos sectores donde la acción del Keuper, en su salida diapírica, no ha sido capaz de traspasar la cobertera jurásica.

Tiene lugar, a continuación, la sedimentación cretácica, con un predominio [184] de los

${ }^{5}$ RODRÍGUEZ ESTRELLA, T.: Geología e hidrología del sector Alcaraz-Lietor-Yeste (Prov. de Albacete). Sintesis geológica de la zona Prebética. I. G. M. E. Madrid, 1971 p. 151.

${ }^{6}$ Memoria del Mapa Tectónico de la Península Ibérica y Baleares. Serv. de Publicaciones del Ministerio de Industria, Madrid, 1977. p. 48.

\footnotetext{
${ }^{7}$ Memoria del Mapa Tectónico de... Op. cit. p. 49

${ }^{8}$ RODRÍGUEZ ESTRELLA, T. Op. cit. p. 151

${ }^{9}$ RODRÍGUEZ ESTRELLA, T. Op. cit. p. 155.
} 
materiales margosos alternantes con bancos margocalizos, y cuyos afloramientos principales se localizan al W según la línea límite Busot-Villafranqueza. El medio de deposición varía de unos pisos a otros, produciéndose a finales del Cretácico la regresión senonense que traslada la línea de costa a la región de Caudete (prov. de Albacete) a partir de la cual se localizaría en la región de Elda-Alicante una amplia plataforma subsidente en un medio de baja energía ${ }^{10}$.

El Cenozoico está representado en la zona de estudio desde el Paleoceno (transgresivo) hasta el Plioceno (de origen continental). Aparecen en él dos dominios paleogeográficos divididos por el accidente del Río Seco que actúa a modo de fractura paleogeográfica. La existencia de esta división de dominios responde a la actuación de los movimientos alpinos que, en el área analizada, empezaron a funcionar a finales de la Era Secundaria (Fase Larámica). Así, el sector $\mathrm{N}$ durante el Paleoceno se presenta bajo el dominio de «un mar somero y poco subsidente con depósitos propios de plataforma interna ${ }^{11}$ y sedimentación de margas y margo-calizas en alternancia flyschoide. Las mismas condiciones de estabilidad se dan en el Eoceno en este sector $\mathrm{N}$ mientras que, por el contrario, al sur del accidente del Seco la mayor potencia de «los depósitos eocenos incita a pensar en una mayor subsidencia» ${ }^{12}$.

En el Oligoceno, ambos dominios paleogeográficos van a presentar comportamientos diferentes ante los empujes que sobre ellos van a ejercer los movimientos medio-alpídicos (Fase Pyreniense) que en estos momentos se dejan sentir. Así, el sector $\mathrm{N}$ experimenta una fuerte subsidencia que permite una importante sedimentación de materiales (Surco Flysch), mientras que el sector meridional aparece en dicho momento emergido.

En el período Helvetiense empiezan a funcionar los movimientos postalpídicos en elárea que produjeron una desnivelación de bloques teniendo como línea de referencia el cauce del Río Seco, provocando el levantamiento del sector septentrional y el hundimiento del meridional «permitiendo al mar mioceno transgredir sobre las formaciones más antiguas» ${ }^{13}$.

Con la retirada del mar mioceno y, en consecuencia, el cese de su sedimentación, el área debió quedar configurada como una cuenca endorreica, cerrada al mar por una serie de umbrales y en la que se van a ir [185] depositando los materiales pliocenos y villafranquienses. Ello explica, hasta cierto punto, la inexistencia de materiales de origen marino posteriores al Mioceno en la zona de estudio.

La transición del Cenozoico al Cuaternario viene marcada en el área analizada por una formación que sirve de nivel guía para enlazar el Plioceno, último período del Terciario, con el Villafranquiense, primera etapa de la Era Cuaternaria ${ }^{14}$.

Este paquete constituye lo que se ha denominado «Formación Sucina» por presentar caracteres litológicos y faunísticos similares a los definidos por MONTENAT en la localidad de Sucina ${ }^{15}$

Posteriormente a la deposición de dicha formación, tiene lugar la actuación de una nueva

${ }^{10}$ LENDÍNEZ GONZÁLEZ y LERET VERDÚ. Memoria del Mapa Geológico de España E 1:50. 000 Hoja $n^{\circ}$ 872(Alicante) I. G. M. E. p. 30.

${ }^{11}$ LENDÍNEZ GONZÁLEZ y LERET VERDÚ. Op. cit. p. 30

${ }^{12}$ LENDÍNEZ GONZÁLEZ y LERET VERDÚ. Op.cit. p. 31.

${ }^{13}$ LENDÍNEZ GONZÁLEZ y LERET VERDÚ. Op. cit. p. 31.

${ }^{14}$ CUENCA PAYÁ, A. y WALKER, M. J.: «Comentarios sobre el Cuaternario en el centro y sur de la provincia de Alicante» en Actas de la I Reunión Nacional del Grupo Español de Trabajo del Cuaternario. Madrid, 8-11, Octubre, 1973 p. 16

${ }^{15}$ LENDÍNEZ GONZÁLEZ y LERET VERDÚ. Op. cit. p. 19 
fase orogénica relacionada con los movimientos postalpídicos que van a producir una serie de líneas de fractura de cierta entidad y, entre ellas, una cuya trayectoria es paralela al curso actual del Río Seco y que originó una línea de debilidad tectónica en el fondo de la cuenca endorreica colmatada por los materiales de la Formación Sucina. Esa línea de fractura sería aprovechada por el Río Montnegre para encauzar su trayectoria hacia el mar y para permitir el desagüe de la cuenca endorreica que, a partir de ese momento, pasará a ser exorreica, canalizándose la escorrentía a través de dicho río y de los barrancos a él adyacentes.

Tectogénesis.- Desde un punto de vista tectónico, cabría señalar en el área de estudio las siguientes unidades:

1) Un zócalo jurásico plegado y fallado que, en ocasiones, por la existencia de fallas inversas y favorecido por un nivel de despegue triásico, hace extrusión rompiendo la cobertera mesozoica, es decir, perforando los materiales cretácicos que culminan la serie secundaria.

2) Materiales cretácicos replegados en la cobertera gracias a los fenómenos de despegue de los niveles incompetentes de la serie litológica.

3) Estructuras giradas, fenómenos de arrastres en fallas y pliegues, en íntima relación con accidentes tectónicos mayores como evidencian las líneas sismotectónicas del Río Seco, Sagunto-Jijona-Alicante, la arista de dislocación alicantina, línea paralela a la costa y que discurre al norte de Campello-Villajoyosa y Altea y los arcos de hundimiento situados en la zona costera, generadores de los óvalos morfotectónicos de Valencia y Alicante, según LENDÍNEZ GONZÁLEZ y LERET VERDÚ.

Todas estas líneas de debilidad tectónica son las que están produciendo [186] una manifiesta actividad sísmica, cuya impronta más notoria y reciente se dejó sentir en 1981 con una sacudida que superó el grado IV en la escala Mercalli.

\section{CLIMATOLOGÍA}

Introducción.- La zona se encuentra enclavada en el amplio conjunto que constituyen las tierras valencianas. Toda una serie de trabajos y tesis doctorales recientes han contribuido, en gran medida, a establecer una delimitación del ámbito mencionado desde un punto de vista estrictamente climático. Atendiendo a dichos estudios, la ubicación del área objeto del presente trabajo se halla enmarcada en la zona de los Llanos Litorales, en su segmento más meridional, el cual se extiende a partir del Cabo de San Antonio, incluyendo los valles bajos del Montnegre, Vinalopó y Segura ${ }^{16}$, donde las condiciones pluviométricas y de balance hídrico cambian sensiblemente con respecto a los segmentos litorales ubicados en una posición más septentrional para entrar, progresivamente, en la región climática del SE peninsular, cuyas características son la indigencia pluviométrica y una elevación media de las temperaturas.

Las precipitaciones.- El régimen pluviométrico que afecta al área es, claramente, mediterráneo con una marcada aridez y una elevada irregularidad interanual. Su rasgo básico es una llamativa indigencia pluviométrica, al igual que acontece en todas las zonas del SE español, concretamente el observatorio-patrón de donde han sido obtenidos los datos (Ciudad-Jardín), registra una precipitación media anual de $347,72 \mathrm{~mm}$ en la serie de treinta años analizada (1950-1979). En dicho período, el 50\% de los totales anuales están próximos a la media, el 33'3\% son inferiores a la misma, mientras que tan sólo tres años superan el umbral de los 500 $\mathrm{mm}$, de estos datos deriva la existencia de una elevada irregularidad interanual.

Por otro lado, hay que tener en cuenta un segundo dato básico y es que esa indigencia de las precipitaciones no es sólo función del total pluviométrico sino también del reducido número

${ }^{16}$ CLAVERO PARICIO, P. L.: Los climas de la región valenciana. Resumen de Tesis doctoral. Univ. de Barcelona, Barcelona, 1980. 
de días con precipitación al cabo del año, que en el ámbito de estudio es de 85’3 (en este dato se recogen también los días con precipitación inapreciable).

Una vez expuestos estos datos relevantes, y antes de establecer cualquier tipo de determinismo climático en base a una caracterización pluviométrica, conviene poner de manifiesto los datos que, posteriormente [187], nos permitirán encuadrar el área de estudio en el seno de una tipología previamente establecida por los estudios de Climatología regional. Estos datos son las precipitaciones medias mensuales cuyos valores son los siguientes:

\section{MES $\quad$ PRECIPITACIÓN (en mm)}

Enero................................................................... 19,

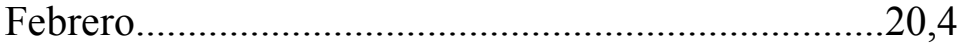

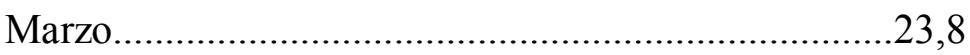

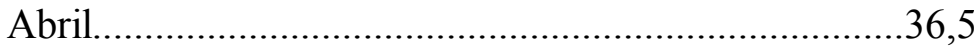

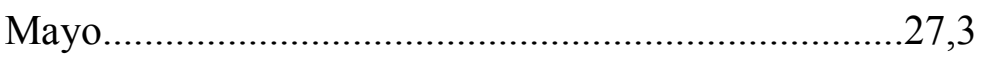

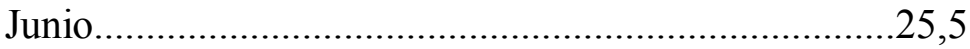

Julio......................................................................... 4,3

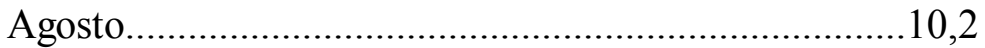

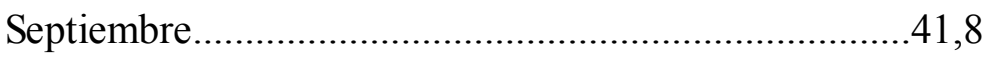

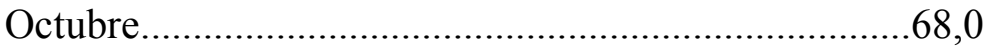

Noviembre................................................................ 36,6

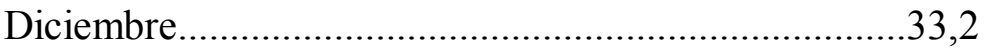

El análisis de estas cifras permite incluir a la zona de estudio dentro del régimen climático mediterráneo con dos máximos equinocciales, siendo preponderante el máximo otoñal, aunque en realidad no puede decirse que el invierno sea absolutamente seco. La aportación otoñal representa el 66,6\% de las precipitaciones anuales, destacando octubre como el mes más lluvioso. En el ritmo de las precipitaciones aparecen, además, dos mínimos: el principal de verano y el secundario de invierno. El primero aporta el 11,4\% de la precipitación anual mientras que el segundo lo hace con el 20,7\%.

La explicación a esta peculiar distribución de las precipitaciones hay que buscarla en un análisis de la dinámica atmosférica que domina el área en la cual se encuentra inmersa la zona de estudio. Las precipitaciones son aquí aportadas en su casi totalidad por la existencia de procesos de gota fría o baja desprendida en altura, mientras que en función de la situación de sotavento del SE peninsular el paso de las borrascas atlánticas apenas si supone unos totales muy reducidos.

Por lo que se refiere a los mecanismos que permiten estos aguaceros de excepcional intensidad horaria, hay que tener en cuenta dos hechos: en primer lugar, la presencia de aire anormalmente frío en altura, lo que provoca brutales exageraciones del gradiente térmico en la vertical y, en segundo lugar, la existencia de aire cálido y húmedo, muy inestable, hecho favorecido por la inercia térmica de las aguas marinas en función [188] de lo cual es en la época otoñal cuando las temperaturas superficiales del agua del mar registran sus más elevados valores 
térmicos.

Hasta aquí hemos descrito los mecanismos que provocan el desencadenamiento de precipitaciones de excepcional intensidad horaria, precipitaciones que pueden definirse como torrenciales y cuyo umbral, LINES ESCARDO, el año 1972, en una comunicación al Coloquio Meteorológico de Lisboa, cifró en no menos de $50 \mathrm{~mm} / 24 \mathrm{~h}$. En la zona de estudio, el observatorio de Ciudad Jardín ofrece una frecuencia media de días con precipitación superior al umbral antes citado de 0,8 veces, es decir, precipitaciones de esa naturaleza se producen una vez cada 15 meses en nuestro ámbito, siendo el total más elevado a lo largo del período de treinta años estudiado el del día 15 de octubre de 1962 con una pluviometría de 133,8 mm y una intensidad de $305 \mathrm{~mm} / 24 \mathrm{~h}$.

Las temperaturas.- Para el estudio del régimen térmico contamos con la misma serie de años utilizada para efectuar el análisis de las precipitaciones (1950-1979). Del manejo de los datos, se obtiene una temperatura media anual de $18^{\circ} \mathrm{C}$, por lo que térmicamente representa un dominio de clima mediterráneo con una irregularidad interanual de tan sólo $2,5^{\circ} \mathrm{C}$.

En cuanto al ritmo anual de las temperaturas, se observa que a partir del mes de agosto las mismas van decreciendo progresivamente hasta enero inclusive, mes a partir del cual se inicia ya una recuperación térmica que se prolonga hasta agosto. En esta ganancia térmica cada mes registra, por término medio, de 1 a $3{ }^{\circ} \mathrm{C}$ más que el mes que le procede, salvo en los casos de julio y agosto, donde el incremento es de tan sólo tres décimas de grado.

Del manejo de los datos térmicos obtenidos se deducen las siguientes conclusiones:

1) Las temperaturas máximas absolutas se registran en los meses de julio y agosto, siendo el primero el que alcanza los valores superiores, prueba de ello lo constituye el día 12 de julio de 1961, en el que se registró una temperatura de $41,2^{\circ} \mathrm{C}$.

2) Las temperaturas mínimas absolutas se dan en los meses de enero y febrero, registrando este último los valores más inferiores. Un dato que ejemplifica este hecho es la increíble temperatura de $-4,6^{\circ} \mathrm{C}$ registrada el día 12 de febrero de 1956.

3) Finalmente, y como tercera consecuencia, cabe señalar a marzo como el mes de mayor amplitud térmica absoluta $\left(23,1^{\circ} \mathrm{C}\right)$. Quizás, la causa de ello esté en que se trata, precisamente, de un mes de transición en el que el invierno no ha perdido todavía su vigencia, pero, a la vez, se está produciendo una ganancia calorífica motivada por una disposición [189] más vertical de los rayos solares, disposición que, naturalmente, redunda en una mayor efectividad de la radiación calorífica.

Se trata, en definitiva, de una zona con un régimen térmico plenamente mediterráneo, caracterizado por un verano relativamente caluroso y por la suavidad del invierno.

La aridez.- Con las premisas antes expuestas, de precipitaciones y temperaturas, es fácil comprobar que el medio que ahora estudiamos se encuadra dentro del dominio de los climas semiáridos.

Con arreglo a la clasificación de Thornthwaite, el clima queda definido como $\mathrm{DB}_{3}{ }_{3} \mathrm{da}$ '. La letra $\mathrm{D}$ es indicativa de un índice hídrico o grado de humedad muy bajo $(-31,119)$ que incluye el área dentro de los climas semiáridos. $\mathrm{B}{ }_{3}$ representa una evapotranspiración propia de un clima Mesotérmico III $(914,28)$ en el que, en función de la eficacia térmica, las precipitaciones están expuestas a un grado de evaporación muy elevado. La minúscula d traduce un exceso de agua pequeño o nulo y, finalmente, a' señala una clara influencia marina al ser su índice de 46,1 sobre la evapotranspiración ya que, lógicamente, las brisas marinas veraniegas disminuyen la eficacia evaporadora de las altas temperaturas.

\section{MORFOLOGÍA SEMIÁRIDA: LOS GLACIS}

Si un tipo de modelado predomina en la zona de estudio éste es el sistema morfogenético 
semiárido, cuya acción se manifiesta de muy diversas formas, pero entre ellas llaman la atención por su singular aspecto las superficies que configuran el piedemonte de las alineaciones montañosas, superficies con unas características peculiares y que han sido objeto de las más variadas denominaciones y que, en el presente trabajo, vamos a analizar bajo la designación de «glacis».

Este tipo de modelado es, como ya hemos dicho, elemento característico de los climas semiáridos y aquí, en el SE de la Península Ibérica, dichas formas ocupan casi la mitad de las llanuras existentes. Tan amplio espacio como el que necesitan los glacis para su configuración es un hecho que requiere largo tiempo. Su amplia difusión permite alcanzar una conclusión: el clima árido se mantiene aquí desde hace mucho tiempo. En la búsqueda de plantas endémicas, FREITAG (1866) llegó a la conclusión de que el SE español presentaba ya en el Mioceno un clima árido. Únicamente en el Plioceno final y en algunas fases del Pleistoceno dominó un clima más suave, pero existieron, en cambio, períodos con mayor aridez que la actual.

Los glacis se presentan en varios niveles, habiendo contabilizado en la Cuenca de Busot hasta cuatro planos inclinados a diferentes alturas, [190] de los cuales el inferior enlaza con el gran glacis que tapiza el Llano de Alicante.

El proceso de formación de los glacis es un tema, en gran medida, polémico por cuanto, en realidad, no se dispone aún de los estudios suficientes capaces de precisar si se trata de un único proceso o si, por el contrario, intervienen mecanismos diferentes en la elaboración del plano inclinado. Así, frente al sistema del «sheet flood»o «arroyada en manto» tradicional, en la actualidad se argumenta como configurador de los glacis la erosión fluvial lateral o el desplazamiento de los lechos o pequeños canales. Así, para DUMAS ${ }^{17}$, el proceso de elaboración de los glacis tiene su origen en los flujos procedentes de los dispositivos topográficos. Se trata de flujos que rebajan la pendiente después que la evacuación haya sido, inicialmente, concentrada en los torrentes que inciden, modestamente, el relieve en la parte superior. Estos torrentes experimentan múltiples desplazamientos en función de toda una serie de cambios de trazado de los canales de evacuación, movilidad ésta que se traduce en la existencia de unos regueros de gravas poco espesos que describen formas zigzagueantes. La evacuación derramada en el eje del canal transporta y deposita las gravas, mientas que la escorrentía difusa en superficie se encarga, únicamente, de movilizar las fracciones finas (limos disponibles). La escorrentía acompaña cada crecida, volviendo a emprender el trabajo abandonado al final del período de escorrentía precedente. Estas paradas sucesivas aseguran el transporte de materiales finos hasta el nivel de base río abajo, además, soportan y recubren los regueros de gravas en zig-zag.

Varias son las causas que aquí concurren para favorecer la configuración de estas formas del modelado. Así, en íntima relación con este proceso de elaboración de los glacis, un hecho que destaca e influye sobremanera en el mismo es el régimen climático, puesto que de la relación existente entre temperaturas y precipitaciones va a derivar un sistema de erosión, extremadamente, potente. Totales anuales reducidos (347,72 $\mathrm{mm}$ de precipitación media anual) es una de las características pluviométricas más relevantes, si bien lo verdaderamente llamativo estriba en la concentración de esos totales en unos pocos días de lluvia al año, tratándose, en consecuencia, de chubascos de fuerte intensidad horaria. A ello se suman unas temperaturas medias elevadas que determinan unos niveles de evaporación muy intensos, sobre todo en la estación estival, por lo que las disponibilidades hídricas en los suelos son muy escasas y, en determinados meses, prácticamente nulas, de ahí que la vegetación natural se vea reducida a poblamientos muy abiertos, [191] tratándose siempre de un matorral cuyos elementos aparecen muy separados entre sí para que cada planta pueda aprovechar mejor la escasa humedad existente en el subsuelo.

${ }^{17}$ DUMAS, B. Le Levant espagnol. La genèse du relief. París 1977 p. 409. 
Otro factor que influye en la denudación de estas vertientes montañosas es la existencia de materiales poco consistentes al tratarse, fundamentalmente, de margas y margo-calizas que son fácilmente erosionables, o bien calizas que, a pesar de ser mucho más resistentes, se encuentran muy tectonizadas, presentando un estado de fisuración y diaclasamiento muy elevado en función de los grandes empujes a que han sido sometidas.

Junto a estas condiciones juegan también un papel muy importante las pendientes que presentan los flancos de donde parten los flujos que configuran los glacis. La Cuenca de Busot presenta una inclinación del terreno con pendientes comprendidas entre los $8^{\circ}$ y los $14^{\circ}$ de pendiente media, si bien cabe señalar que las de las laderas montañosas son muy superiores, así por ejemplo, la Sierra de Venta presenta una inclinación desde la culminación hasta el «knick» de los glacis de unos $45^{\circ}$ y las de la Sierra de Bonalba giran en torno a los $23^{\circ}$.

El factor inclinación del terreno es de una importancia trascendental en los arrastres ocasionados por el agua de escorrentía, puesto que cuanto mayor es el gradiente de inclinación mayor es la velocidad del agua que circula sobre ellos, haciendo así que sea mayor la cantidad de suelo arrastrado. Igualmente, la velocidad del agua se va incrementando conforme desciende por la ladera, de ahí que un terreno inclinado pierda más suelo en la parte inferior del mismo que en la superior.

Cabría analizar ahora las causas que han motivado el modelado de la Cuenca de Busot a base de cuatro superficies de erosión, sin duda relacionadas con variaciones verticales del nivel de base local en función del tiempo, variaciones que para DUMAS constituyen el primer factor de formación de los glacis. Así, para el nivel superior o 1 esa variación posee un carácter estructural, mientras que para los niveles 2, 3 y 4 es de origen climático. El glacis superior tuvo su nivel de base en lo que constituyó la antigua cuenca endorreica plio-villafranquiense, cuyos restos principales se encuentran hoy en la parte Oeste de la carretera de Villafranqueza a Tángel y en el piedemonte de la Sierra de Boter. El paquete villafranquiense se halla recubierto por depósitos de glacis del Cuaternario antiguo y todo el conjunto se encuentra basculado hacia el Llano de Alicante, lo que pone de manifiesto la actuación de la neotectónica durante las primeras etapas del Cuaternario, que provocó el levantamiento de los bordes de la antigua cuenca endorreica y el hundimiento de su parte central, a la vez que se producía el resquebrajamiento de la misma con la aparición de una línea de fractura a través de la cual se [192] efectuó el desagüe de la cuenca lacustre y la posterior canalización de la escorrentía hacia el mar, constituyendo una especie de golfo que se inundaba en los períodos cálidos del Cuaternario o que permanecía vacío en las épocas regresionales o frías, variando, de esta manera, el nivel de base general, siendo en función de estas fluctuaciones glacio-eustáticas como pudieron ir desarrollándose los depósitos glacificados de los estadios 2, 3 y 4.

Nivel de glacis superior.- Se encuentra, en su parte más elevada, a la altitud de $320 \mathrm{~m}$ s.n.m. De él quedan únicamente una serie de restos que permiten, fácilmente, su reconocimiento, y que aparecen diseminados a lo largo de toda la cuenca, siendo los ejemplos más significativos la serie de elevaciones próximas al pueblo de Busot, donde en la actualidad se han construido grandes embalses para el almacenamiento de aguas para riego y aquellos otros ubicados en la margen derecha de la carretera de Busot a Jijona a los 2,5 Km del cruce. Otros restos importantes son los que se encuentran entre el Barranco de Vercheret y la carretera de Jijona (N-340) y entre dicho barranco y el de Agua Amarga, si bien se trata de restos de dimensiones inferiores pero con la suficiente potencia para frenar el desarrollo de los niveles 3 y 4 que parten de la Sierra de Venta y que han visto limitada su expansión en función de la existencia de estos relieves residuales, testigo de la primera superficie de erosión. Este primer nivel de glacis enlaza con el gran glacis de San Vicente, próximo a dicha localidad, y cuya terminación meridional se encuentra en el lugar conocido como La Rabasa.

Este nivel más antiguo lo hemos datado como Villafranquiense, pues su nivel de base 
coincide hoy con lo que fue el fondo de la antigua cuenca endorreica, configurada en los albores del Cuaternario. Estratigráficamente presenta las siguientes capas en orden inverso a su deposición:

-Costra calcárea cementada en hojas $(50 \mathrm{~cm})$

-Estrato 1a.- Cantos heterométricos y graves envueltos en matriz intersticial de textura areno-arcillosa.

-Estrato 1b.- Depósito compuesto, básicamente, por arcillas y limos que engloban una débil fracción gruesa (0’37\%).

-Estrato 1c.- Acumulación de material grueso, con cantos en ocasiones de gran tamaño y con una débil proporción de fracción fina $(21,3 \%)$.

-Estrato 1d.- Compuesto en un porcentaje superior al 70\% por arcillas, arenas y limos entre los que se entremezclan pequeños cantos de dimensiones entre $2-3 \mathrm{~cm}$ en su eje mayor y nódulos de cal.

-Estrato 1e.- Básicamente integrado por elementos de la fracción [193] gruesa y de la fracción grava $(76,1 \%)$ envueltos en una matriz de textura francamente arenosa.

\section{GRÁFICO 1}

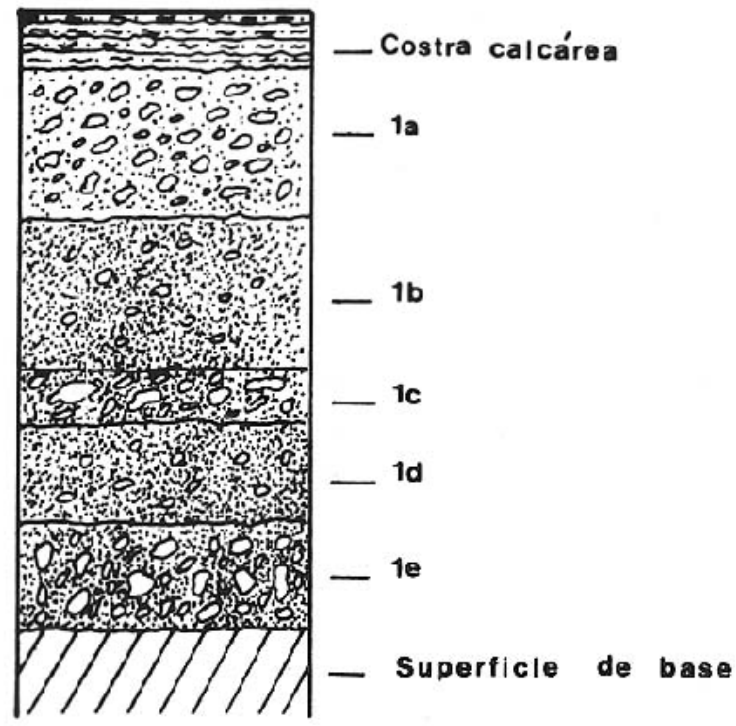

La costra calcárea que recubre este primer nivel de glacis es de las denominadas por RUELLAN $^{18}$ «encostramientos hojosos en losas compactas» con hojas duras de 5 a $10 \mathrm{~cm}$ de espesor, separadas entre sí, petrificadas y continuas, conteniendo un $80 \%$ de $\mathrm{CaCO}_{3}$, se encuentra [194] en gran parte desmantelada por la acción antrópica y por la disolución kárstica.

Segundo nivel de glacis.- Se presenta, al igual que el anterior, en una serie de restos que se extienden por toda la Cuenca de Busot. Así, encontramos retazos de este segundo nivel en las siguientes localizaciones:

1) A $\operatorname{los} 2,5 \mathrm{Km}$ del cruce de la carretera de Busot a Jijona, próximo a los restos del glacis superior.

2) En las cercanías de la casa de la Alquedra.

3) A lo largo de todo el piedemonte W de la Sierra de Bonalba, bordeando al Barranco

${ }^{18}$ GIGNOUX, M. y FALLOT, P.: «Contributión à la conaissance des terrains néogènes et quaternaires marins sur les côtes mediterranéennes d'Espagne» C.R. du XIV Congrés Géol. Intern. (1926), fasc. 2 pp. 413-442. 
de Agua Amarga por su margen derecha e izquierda.

\section{GRÁFICO 2}

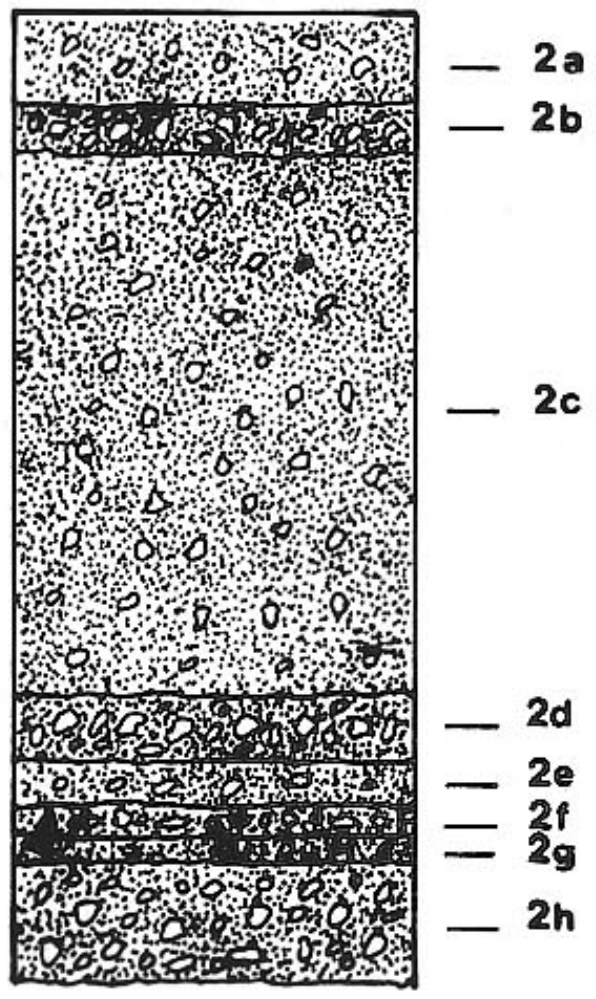

\section{[195]}

Aparece, también, disecado por múltiples torrentes que han permitido el afloramiento de la superficie de base cretácica. Este segundo nivel se encuentra en su parte más elevada a la altitud de $285 \mathrm{~m}$ sobre el nivel del mar, es decir, que el desnivel existente entre el primer estadio de glacis y el que ahora nos ocupa es de unos $35 \mathrm{~m}$. La estratigrafía que presenta es la siguiente, de techo a muro:

-Estrato 2a.- Fundamentalmente compuesto por materiales de la fracción fina (arenas, limos y arcillas) englobando algunos cantos y gravas.

-Estrato 2b.- Integrado por material grueso, con cantos de dimensiones variables pero que nunca superan los $6 \mathrm{~cm}$ de longitud en su eje mayor.

-Estrato 2c.- Compuesto, básicamente, por limos, arcillas y arenas, englobando algún canto de pequeño tamaño.

-Estrato 2d.- Capa de materiales gruesos con matriz intersticial.

-Estrato 2e.- Estrato fundamentalmente integrado por materiales de la fracción fina, con ausencia total de cantos y débil proporción de gravas.

-2f.- Acumulación de arenas, limos y arcillas con escasos cantos y gravas.

-Estrato 2g.- Similar al anterior, pero con una mayor abundancia de materiales de la fracción gruesa y de la grava.

-Estrato 2h.- Material heterométrico, con abundantes cantos y gravas, englobados en una matriz areno-arcillosa. 
La costra calcárea se encuentra hoy en gran parte desmantelada por la acción antrópica, ya que este segundo nivel ha sido objeto de una importante actividad agrícola.

A la hora de establecer una datación, resulta obvio que este nivel debió configurarse en el Pleistoceno medio, puesto que el nivel superior es villafranquiense y el tercer estadio ha sido datado como würmiense por DUMAS ${ }^{19}$, por consiguiente este segundo nivel posee una edad que data de los períodos Mindel o Riss.

Los glacis de Venta: niveles 3 y 4.- En el piedemonte sur de la alineación montañosa de Venta, eje que constituye el límite más occidental de la zona de estudio, en el marco de la Cuenca de Busot, aparecen, perfectamente definidos, los niveles 3 y 4 . Es precisamente en esta zona donde la apariencia de glacis se deja sentir con mayor intensidad, puesto que los niveles anteriores se encuentran muy recortados, apareciendo, como ya se ha dicho, únicamente lambeaux de glacis. Sin $\mathrm{em}^{20}{ }^{20}[196]$

Los depósitos glacificados que franquean por el sur el relieve monoclinal de Venta poseen un eje longitudinal que se extiende sobre más de $1,5 \mathrm{Km}$ y la línea donde se produce el contacto entre el relieve estructural y el deposicional, o sea, el Knick, se encuentra a una altitud de $300 \mathrm{~m}$ sobre el nivel del mar. El desarrollo longitudinal de los mismos aparece limitado hacia la parte baja por la presencia del Barranco de Vercheret, eje de drenaje que toma una dirección casi paralela a la del escarpamiento monoclinal de Venta, produciéndose el contacto entre los glacis y dicho barranco a través de una terraza engendrada por el mismo a la altura de $210 \mathrm{~m}$ sobre el nivel del mar.

Como ya se ha dicho, son dos los niveles que aparecen al pie de este escarpamiento montañoso. La segunda superficie glacificada de Venta se presenta claramente encajada entre los restos del tercer nivel, del cual sólo quedan escasos retazos, si bien son fácilmente reconocibles por su superficie allanada y su ligera pendiente.

Este nivel presenta en la sucesión estratigráfica las siguientes capas de techo a muro:

\section{GRÁFICO 3}

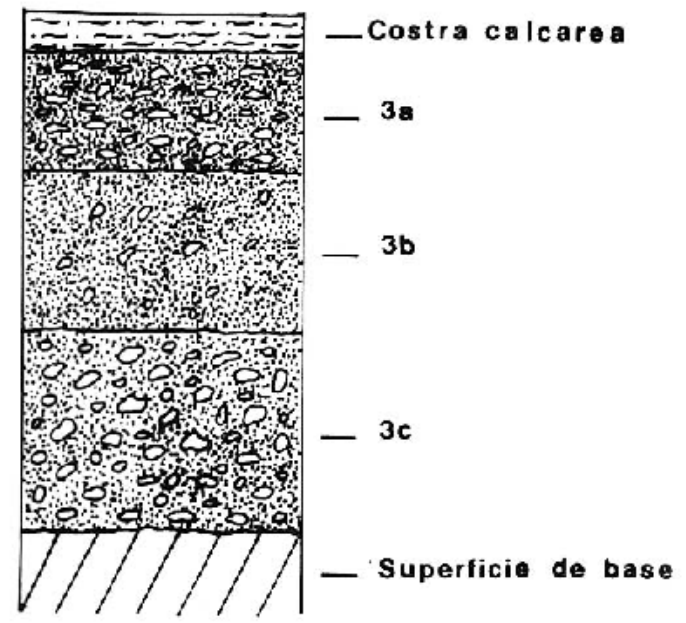

[197]

\footnotetext{
${ }^{19}$ DUMAS, B. Op. cit. p. 408.

${ }^{20}$ [Esta página se corta aquí (N. del E.)]
} 
-Estrato 3a.- Costra calcárea poco cementada.

-Estrato 3b.- Estrato gravoso con pequeños cantos.

-Estrato 3c.- Estrato limo-arcilloso.

-Estrato 3d.- Estrato de gravas heterométricas.

-Estrato 3e.- Superficie de base cretácica.

GRÁFICO 4

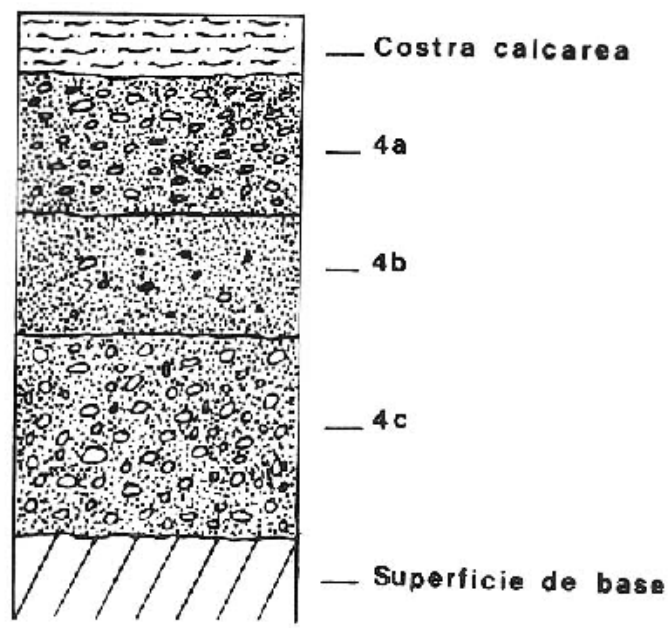

El cuarto y último nivel presenta las siguientes capas:

-Estrato 4a.- Costra calcárea poco cementada.

-Estrato 4b.- Estrato de gravas con matriz limo-arenosa.

-Estrato 4c.- Estrato limo-arenoso.

-Estrato 4d.- Gravas heterométricas con matriz limo-arcillosa.

-Estrato 4e.- Superficie de base cretácica.

Ambos niveles se hallan recubiertos por un encostramiento calcáreo pulverulento cuyo espesor es de 30 a $50 \mathrm{~cm}$, sin losas compactas que se desmoronan fácilmente bajo una débil acción mecánica. El cuarto nivel ha sido objeto de un intenso aprovechamiento agrario que ha provocado [198] el desmantelamiento de la costra, si bien, todavía, subsisten algunos restos de la misma en aquellos sectores donde la actividad humana no ha ejercido aún su influencia.

Igualmente, puede observarse un hecho significativo que se materializa en el grado de redondeo de los cantos, así éstos en las partes superiores del glacis presentan una esquistosidad notable, es decir, se trata de cantos con aristas bastante bien perfiladas, ya que su rodamiento ha sido escaso antes de la deposición, mientras que, por el contrario, estos cantos aparecen mucho más desgastados en las partes terminales del glacis, es decir, en las proximidades del nivel de base local en función del cual se han configurado estos planos inclinados, puesto que han tenido que soportar un desplazamiento mayor que ha provocado el hecho de que por el rozamiento estos cantos presenten unas aristas mucho más rebajadas.

En cuanto a las dataciones se refiere, DUMAS señala que ambos glacis poseen una cronología würmiense, perteneciendo el $4^{\circ}$ nivel al Würm reciente, por lo que dicha superficie enlazaría con el que tapiza el Llano de Alicante. 


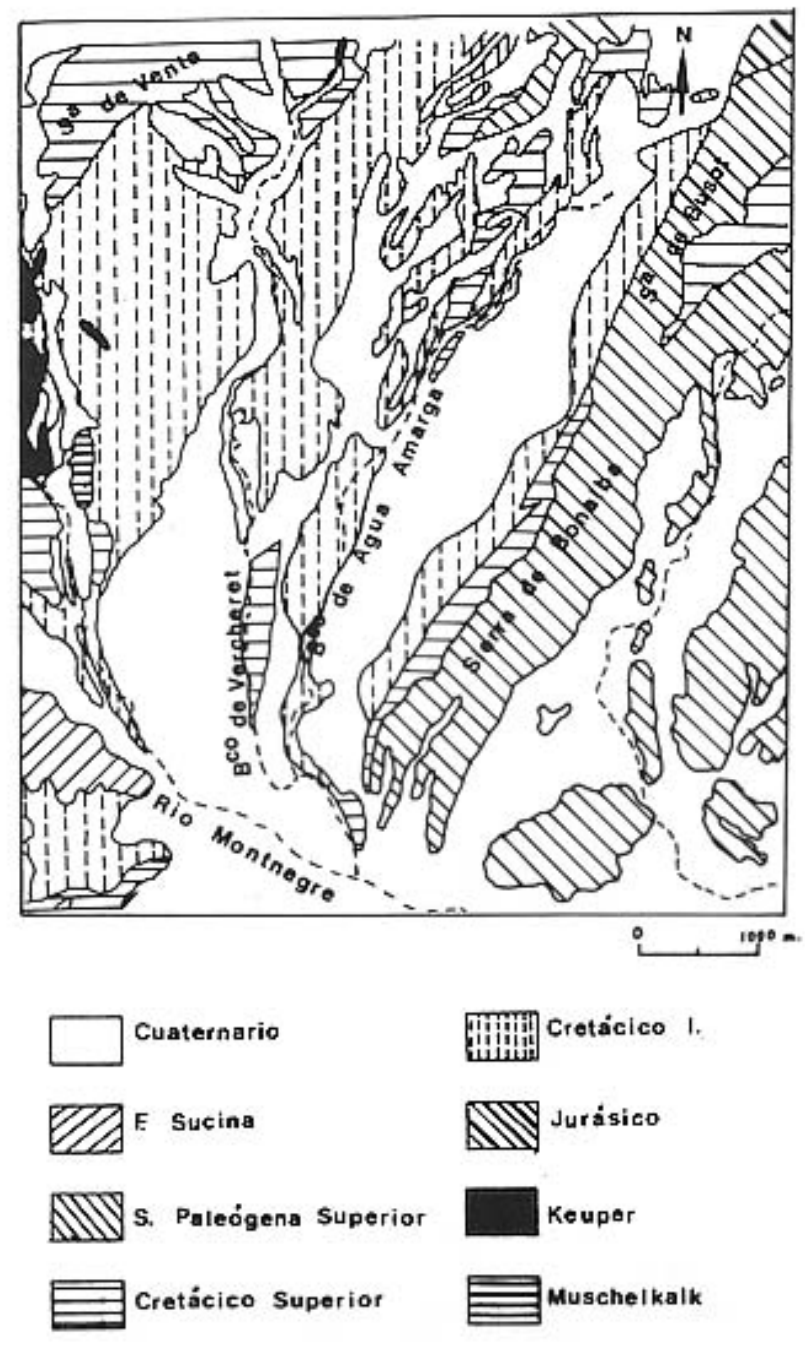




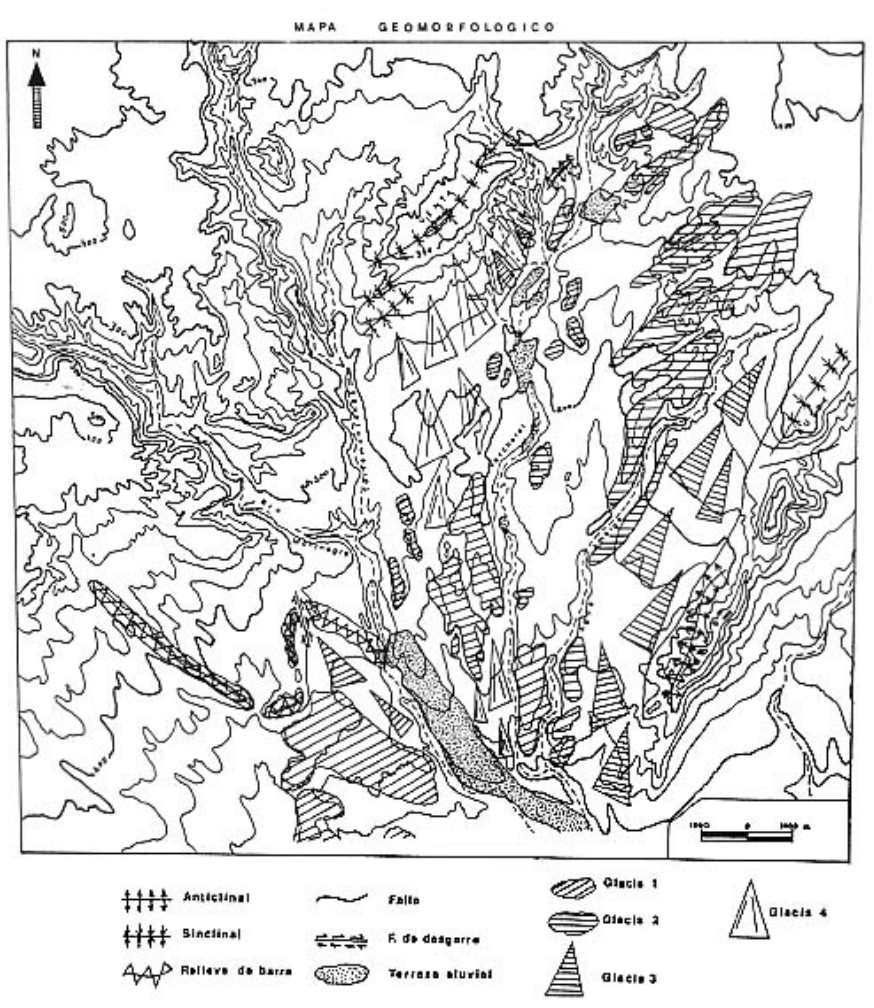

XIII 